\title{
Prenatal diagnosis of sex chromosomal inversion, translocation and deletion
}

\author{
LIN ZHANG, MEIHONG REN, GUINING SONG, YANG ZHANG, \\ XUEXIA LIU, XIAOHONG ZHANG and JIANLIU WANG
}

\author{
Prenatal Diagnosis Center, People's Hospital of Peking University, Beijing 100044, P.R. China
}

Received June 24, 2016; Accepted April 27, 2017

DOI: $10.3892 / \mathrm{mmr} .2017 .8198$

\begin{abstract}
The aim of the present study was to perform comprehensive prenatal diagnosis using various detection techniques on a fetus in a high-risk pregnant woman, and to provide genetic counseling for the patient and her family so as to avoid birth defects. The routine karyotype analysis via amniocentesis, fluorescence in situ hybridization, and whole genome microarray technique were performed for the prenatal diagnosis of the fetus. The fetal karyotype was 46,X,ish der(X) $\operatorname{inv}(X)(p 22.3 q 28) t(X ; Y)(q 28 ; q 11.2)(X Y q t e r+, S R Y-, D X Z 1+$, RP11-64L19+,STS+,XYpter+); namely, one fetal X chromosome belonged to the derivative imbalanced chromosome and this chromosome demonstrated complex chromosomal rearrangements involving inversion, translocation and deletion. Notably, pericentric inversion between Xp22.3 and Xq28 was identified, and the chromosomal microarray technique confirmed that the long arm q28 of the derivative X chromosome had a 1.241-Mb deletion in Xq28, which included Online Mendelian Inheritance in Man genes such as coagulation factor VIII, glucose-6-phosphate dehydrogenase, inhibitor of nuclear factor- $\kappa \mathrm{B}$ kinase subunit $\gamma$, trimethyllysine hydroxylase $\varepsilon$, Ras-related protein Rab-39B and chloride intracellular channel 2. In addition, this chromosome also exhibited the local translocation of fragment Yq11.21-q11.23, which did not include the sex determining region $\mathrm{Y}$ gene. This fetus demonstrated deletion, inversion and translocation syndrome, and may exhibit the corresponding clinical phenotypes (e.g., intellectual disability or general delayed development) (1) of such chromosome abnormalities after birth. Therefore, in prenatal diagnosis, a variety of genetic diagnostic techniques should be comprehensively used based on specific clinical situations, which may accurately reveal the nature, sources and manifestations of the derivative chromosome abnormalities and avoid the birth of children with defects.
\end{abstract}

Correspondence to: Dr Jianliu Wang, Prenatal Diagnosis Center, People's Hospital of Peking University, 11 South Main Street, Xicheng, Xizhimen, Beijing 100044, P.R. China

E-mail: jianliuwangdoc@163.com

Key words: prenatal diagnosis, inversion, translocation, deletion

\section{Introduction}

The routine karyotype analysis is the gold standard method currently used for the detection of fetal chromosomal number and structural abnormalities; however, this method has certain limitations, such as limited resolution, which may result in chromosomal structural abnormalities $<5 \mathrm{Mb}$ not being diagnosed (1-4). The fluorescence in situ hybridization (FISH) technique sets the probe according to the target chromosomal fragment and detects whether the number of chromosomes or the particular gene(s) is/are abnormal or not. FISH has proven invaluable as an ancillary technique for the identification of clinically significant chromosomal aberrations, as FISH can be performed on metaphase or non-dividing interphase cells, and can detect genomic abnormalities with a resolution from 200 to $1,000 \mathrm{~kb}$, depending on the probe size. In genetic diagnosis, FISH enables the rapid detection of relatively low-level mosaicism in cells and FISH may facilitate the characterization of complex chromosome rearrangements identified by G-banded analysis. Despite numerous advantages, FISH does not provide genome-wide analyses and must be targeted to selected genomic regions believed to be of interest in a given case. When the chromosomal type(s) is/are unclear, the FISH results are often difficult to analyze. Furthermore, FISH only detects the chromosomal structural abnormality in the local regions covered by the detection probes. FISH may yield false negative results in cases where genomic imbalances are smaller than the size of a FISH probe or chromosomal rearrangements are complex, or false positive results when two fluorescent signals co-localize due to viewing a three-dimensional nucleus in two dimensions. The advent of the whole genome microarray technique, including comparative genomic hybridization (CGH) and single nucleotide polymorphism (SNP), has enabled the detection of submicroscopic copy number variations of clinical significance, e.g., small genomic deletions and duplications, known as copy-number variants, that are not routinely observed in karyotype analysis $(5,6)$. SNP arrays can also detect genomic regions with loss of heterozygosity (LOH) (6-8). Microarray analysis eliminates the need for dividing cells and can be performed on direct (uncultured) specimens to provide a more accurate assessment of abnormalities. However, the whole genome microarray technique cannot recognize the different types of chromosomal structural abnormalities, including chromosomal inversion, translocation or insertion. 
Furthermore, the precise physical location of genomic gains cannot be determined by microarray analysis and requires G-banding or FISH for further characterization. Therefore, prenatal diagnosis should be based on the characteristics of different cases and use comprehensive diagnostic methods in order to obtain more accurate diagnostic results. The present study reports a case of complex inversion, translocation and deletion, as diagnosed by such integrated prenatal diagnostic techniques as amniotic fluid-karyotype analysis, FISH, and whole genome microarray. In addition, the etiology and clinical manifestations were investigated.

\section{Materials and methods}

General information. The female patient (age, 27 years), experienced regular menstruation (4 days/30 days), gravida 1 para 0 , and had decorated her house prior to pregnancy and during early pregnancy, thus she may have been exposed to hazardous chemicals in her home environment. The maternal serum screening at 16 weeks and 5 days of gestation showed the risk of Down's syndrome value as 1:261. The noninvasive prenatal testing of the maternal peripheral blood at 18 weeks and 4 days of gestation showed aneusomic fetal chromosomes, suggesting suspicious abnormalities of the fetal sex chromosomes. The patient attended the Prenatal Diagnosis Center of the People's Hospital of Peking University (Beijing, China) at 21 weeks and 1 day of gestation for amniocentesis. After the patient provided written informed consent, $20 \mathrm{ml}$ amniotic fluid was sampled under ultrasound guidance for routine amniotic fluid cell culture, karyotype analysis and the corresponding FISH assays. The results indicated that the fetal sex chromosome exhibited inversion and translocation, but the existence of fragment deletion or duplication could not be ruled out, so further assays were recommended. The current study was conducted in accordance with the declaration of Helsinki and with approval from the Ethics Committee of Peking University. Amniotic fluid $(10 \mathrm{ml})$ was sampled again at 25 weeks of gestation for the whole genome microarray assay. The results confirmed the existence of the inversion and deletion in one $\mathrm{X}$ chromosome and partial fragment translocation in the $\mathrm{Y}$ chromosome. The patient and her family requested to terminate the pregnancy, so an induction delivery was performed in the at 27 weeks and 4 days of gestation. The couple was non-consanguineous and had no family history of genetic disease. There was no history of contact with hazardous substances, although the couple had performed home renovations prior to and in the early stages of pregnancy. The karyotype analysis of the peripheral blood of this couple identified the karyotypes of the husband and wife as $46, X Y$ and $46, X X$, respectively.

Amniotic fluid cell culture and karyotype analysis. Amniotic fluid $(20 \mathrm{ml})$ was obtained (with the first $1-2 \mathrm{ml}$ amniotic fluid discarded) and injected into two disposable sterile centrifuge tubes for the centrifugation at $190 \mathrm{xg}$ for $10 \mathrm{~min}$; the supernatant was subsequently discarded, and 2.5-3.0 ml cell suspension was inoculated into $5 \mathrm{ml}$ of each of Gibco Amniomax-II (Thermo Fisher Scientific, Inc., Waltham, MA, USA) and BIO-AMF-2 (Biological Industries, Kibbutz Beit-Haemek, Israel) amniotic fluid media, under sterile conditions, for 6 7-day static culture at $37^{\circ} \mathrm{C}$ and $5 \% \mathrm{CO}_{2}$. The cell growth was observed every day after the medium was changed. When the amniotic fluid cells adhered to the wall and grew vigorously, and the cells in metakinesis exhibited multiple clones under an inverted microscope, the amniotic fluid chromosomes in each culture bottle were collected separately and the slides produced as follows: Colcemid solution (Thermo Fisher Scientific, Inc.) was added to the culture bottle and incubated for a further $2 \mathrm{~h}$ at $37^{\circ} \mathrm{C}$. The medium was removed from the culture bottle and saved in a prelabeled centrifuge tube. Trypsin-EDTA $(1 \mathrm{ml})$ was added to the bottle and the cells washed by tilting the bottle from side to side. The solution was removed from the bottle and $1.5 \mathrm{ml}$ of fresh trypsin-EDTA solution added and the cells bathed thoroughly with the solution by tilting the bottle. The cells were incubated at $37^{\circ} \mathrm{C}$ for $5 \mathrm{~min}$ and then added to the contents of the centrifuge tube and mixed prior to centrifugation at $190 \mathrm{x} \mathrm{g}$ for $10 \mathrm{~min}$. The supernatant was removed and $5 \mathrm{ml}$ of potassium chloride hypotonic solution $0.075 \mathrm{~mol} / \mathrm{l}$ added to the cell, resuspended and incubated for 10-15 $\mathrm{min}$ in a water bath at $37^{\circ} \mathrm{C}$. Freshly made fixative was added to each tube and mixed gently by inverting the tubes twice then centrifuged at $190 \mathrm{x} \mathrm{g}$ for $10 \mathrm{~min}$ and the supernatant discarded and the fixative step repeated an additional three times. Then the cells were suspended in a small volume of fixative to give a slightly opaque suspension and 3 to 4 drops were placed evenly on a cold wet slide an allow to dry. G-band staining (plus C-band staining when necessary) was used to prepare the chromosome specimens, and, in accordance with the International System for Human Cytogenomic Nomenclature (2013) (9), each specimen was analyzed 30 well-dispersed moderately-long metakinesis phases under a light microscope. When the chimera or abnormal karyotype was identified, the analysis was performed for a total of 100 mitotic phases.

FISH analysis. In order to determine the complex chromosome rearrangements and chromosome breakpoints, FISH analysis was performed on the fetus using the locus probes of XYpter/XYqter, DXZ1, STS, RP11-64L19 and SRY (Vysis; Abbott Molecular, Abbott Park, IL, USA), according to the manufacturer's instructions. Human chromosomes were stained by 4'-6-diamidino-2-phenylindole (DAPI) in the dark for 10-15 $\mathrm{min}$ at room temperature and exhibited bright fluorescence at secondary constriction regions of chromosomes 1,9 and 16 , the proximal short arm of 15 , and the distal long arm of Y.

Affymetrix CytoScan $750 \mathrm{~K}$ array. Genomic DNA was extracted from $10 \mathrm{ml}$ amniotic fluid using a commercially available Genomic DNA Extraction kit (QIAamp DNA Blood Mini kit; Qiagen GmBH, Hilden, Germany) according to the manufacturer's instructions. The standard experimental procedure incorporated the following: Digestion, ligation, polymerase chain reaction (PCR), PCR purification, fragmentation, labeling, hybridization, washing, staining and scanning. A microarray (Affymetrix CytoScan 750K Array) was used to detect copy number variants (losses or gains of chromosome material). This platform includes 25 -mer oligonucleotide probes covering the entire human genome with an overall mean probe spacing of $4 \mathrm{~kb}$. Following hybridization, the laser scanner (GeneChip ${ }^{\circledR} 3000$ Scanner with 7G upgrade) was used for scanning the arrays, and the images 


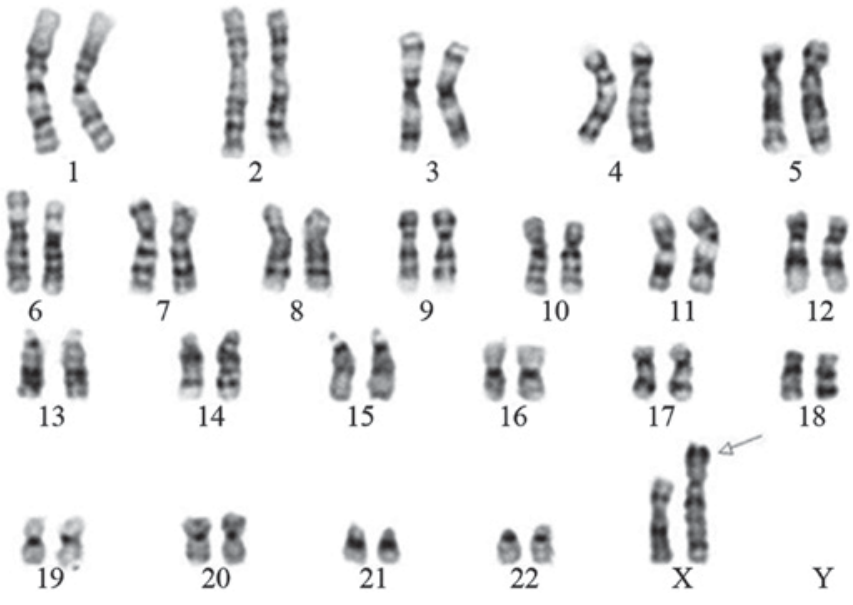

Figure 1. Fetal G-banding karyotype: 46,X,der(X) inv(X) (p22.3q28) $t(X ; Y)$. The fetus had 46 chromosomes and the sex chromosomes were two $\mathrm{X}$ chromosomes, one of which was abnormal. This abnormal $\mathrm{X}$ chromosome exhibited pericentric inversion between Xp22.3 and Xq28. In addition, the short arm of the abnormal $\mathrm{X}$ chromosome exhibited fragment translocation of the $\mathrm{Y}$ chromosome.

were extracted and analyzed using Affymetrix GeneChip Command Console software (version 4.0) and Chromosome analysis software (Chromosome Analysis Suite version 2.1) (both from Affymetrix; Thermo Fisher Scientific, Inc.), respectively.

The Affymetrix CytoScan 750K Array includes 550,000 non-polymorphic markers and 200,000 gene-centric single-nucleotide polymorphisms (SNPs), which enables cytogeneticists to detect and analyze relevant chromosomal aberrations with confidence. This solution provides high-resolution coverage of cancer and constitutional genes of interest, along with high-density SNP coverage for loss of heterozygosity and uniparental disomy detection.

\section{Results}

Karyotype analysis. The routine G-band staining analysis indicated that the fetus had 46 chromosomes; the sex chromosomes were two $\mathrm{X}$ chromosomes, of which one was normal while the other was abnormal. This abnormal $\mathrm{X}$ chromosome may have been due to a pericentric inversion occurring between Xp22.3 and Xq28. In addition, the short arm of the suspicious $\mathrm{X}$ chromosome exhibited fragment translocation of the Y chromosome (Fig. 1).

FISH. The DAPI staining results indicate that the short arm of the abnormal X chromosome exhibited blue fluorescence, which corresponded with the staining characteristics of the long arm of the $\mathrm{Y}$ chromosome; therefore, it confirmed the results of the karyotype analysis that this abnormal $\mathrm{X}$ chromosome exhibited the translocated long arm segment of the Y chromosome (Fig. 2A). The results of FISH using the XYpter (green)/XYqter (red) and STS (red) probe showed that the red fluorescent signal of Xqter, which should appear at the end of the chromosomal long arm, appeared at the end of the short arm of the abnormal X chromosome, but the positive
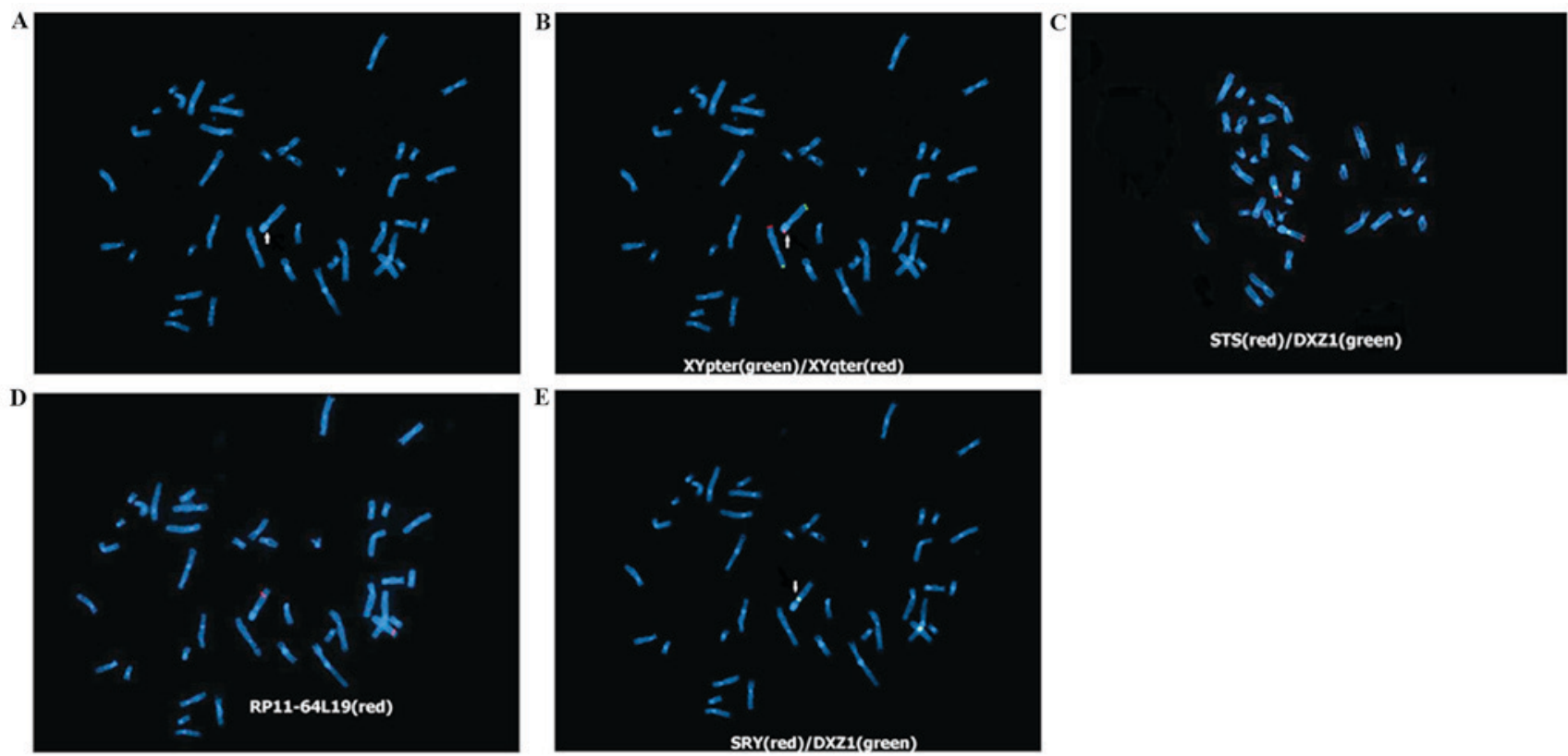

Figure 2. DAPI staining results (magnification, x1,000). (A) Fetal chromosomal DAPI staining. The arrow indicates the short arm of the derivative chromosome X with blue fluorescence, confirming it exhibited the translocated long arm fragment of the Y chromosome. (B) FISH assay using XYpter (green)/XYqter (red) probes. The arrow indicates the red fluorescent signal (XYqter) appeared at the end of the X-chromosomal short arm, while the green fluorescent signal (XYpter) appeared at the end of the long arm, confirming pericentric inversion in this X chromosome. (C) FISH assay using DXZ1 (green) and STS (red) probes. The positive signal of the STS probe appeared at the end of the abnormal X-chromosomal long arm, reconfirming that this abnormal X chromosome exhibited pericentric inversion. The positive green fluorescent signal of the DXZ1probe confirmed that this centromere was on the X chromosome. (D) FISH assay using a RP11-64L19 (red) probe. The positive signal of the RP11-64L19 (red) probe appeared at the end of the abnormal X-chromosomal long arm, indicating that the breakpoint of the long arm inversion should be located before this site. (E) FISH assay using the SRY (red)/DXZ1 (green) and STS (red) probes. The red fluorescence signal of the male testis-determining factor was negative SRY (-). The arrow indicates the positive green fluorescent signal of the DXZ1probe, namely the derivative chromosome was an X chromosome. DAPI, 4-6-diamidino-2-phenylindole; FISH, fluorescence in situ hybridization. 

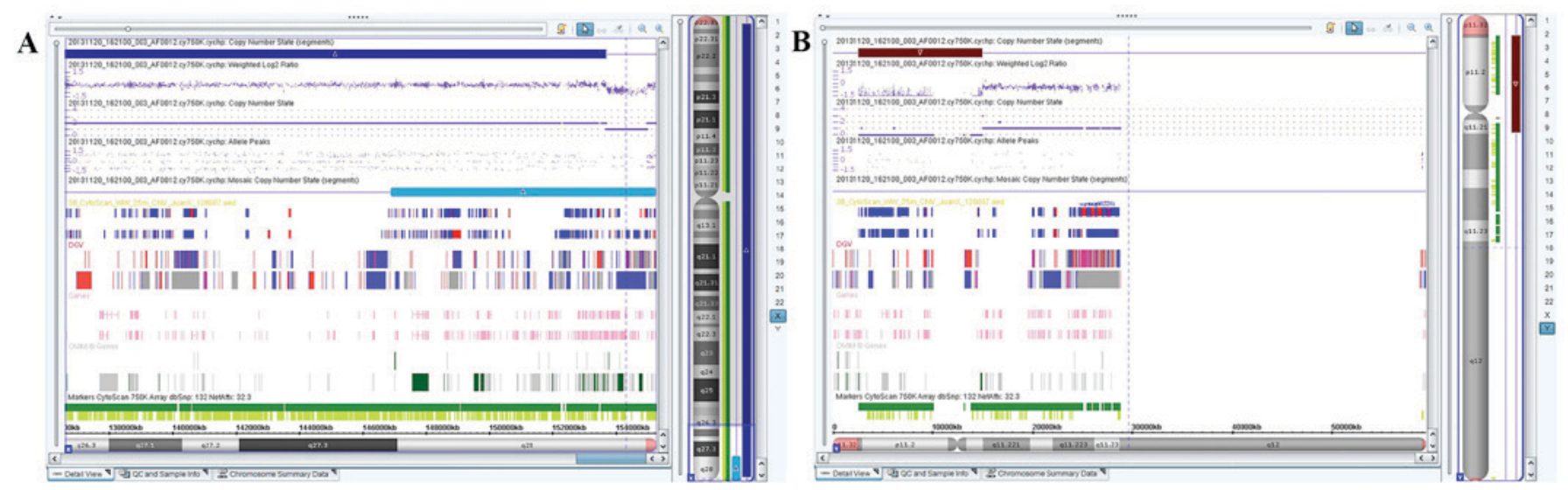

Figure 3. Results of single nucleotide polymorphism microarray assay.arr [hg19]Xq28 (153,689,031-154,930,045) x1, Yq11.21q11.23 (13,979,645-27,799,653) $\mathrm{x} 1$. (A) The $\mathrm{X}$ chromosome; the right inferior region exhibiting a local microdeletion. The long arm of the derivative $\mathrm{X}$ chromosome demonstrated the deletion of a1.241-Mb fragment in q28. (B) The Y chromosome; the region contained a 12.329-Mb fragment between Yq11.21 and q11.23.

signals of Xpter and STS at the end of the X-chromosomal short arm appeared at the end of the long arm of the abnormal $\mathrm{X}$ chromosome. The results confirmed that the abnormal X chromosome exhibited pericentric inversion. Further FISH using the RP11-64L19 (red) probe (located in Xq28) demonstrated that the positive signal appeared at the end of the long arm of the abnormal X chromosome, indicating that the breakpoint of the long arm inversion should be located before this site. Therefore, the $\mathrm{X}$ chromosome exhibited pericentric inversion between Xp22.3 and Xq28. Additional FISH using SRY (red) and DXZ1 (green) probes on the abnormal X chromosome confirmed that this abnormal $\mathrm{X}$ chromosome was SRY-negative, indicating that the translocated Y chromosome was only in the partial long arm of the $\mathrm{Y}$ chromosome rather than the segment that contains the sex-determining region $\mathrm{Y}$ (testis-determining factor) gene in the short arm. The positive green fluorescence of DXZ1 indicated that the centromere was from the $\mathrm{X}$ chromosome, namely the abnormal chromosome was the $\mathrm{X}$ chromosome, namely ish $\operatorname{der}(\mathrm{X}) \operatorname{inv}(\mathrm{X})(\mathrm{p} 22.3 \mathrm{q} 28) \mathrm{t}(\mathrm{X} ; \mathrm{Y})(\mathrm{q} 28 ; \mathrm{q} 11.23)$ (SRY-, DXZ1+, XYpter+, XYqter+, STS+, RP11-64L19+); therefore, the fetal karyotype was $46, \mathrm{X}$, ish $\operatorname{der}(\mathrm{X}) \operatorname{inv}(\mathrm{X})(\mathrm{p} 22.3 \mathrm{q} 28) \mathrm{t}(\mathrm{X} ; \mathrm{Y})$ (q28;q11.23) (XYqter+, SRY-, DXZ1+, RP11-64L19+, STS+, XYptert) (Fig. 2B-E).

SNP microarray assay. The array analysis performed on the DNA extracted from amniotic fluid revealed a $1.241-\mathrm{Mb}$ deletion involving the chromosome region Xq28 and a 12.329-Mb gain involving chromosome region Yq11.21q11.23, or arr[hg19] Xq28(153,689,031-154,930,045) x1, Yq11.21q11.23 (13,979,645 $-27,799,653) \times 1$ (Fig. 3). The results of the SNP microarray assay confirmed that the long arm of the newly derivative $\mathrm{X}$ chromosome contained a deletion of one $1.241-\mathrm{Mb}$ fragment within q28, which contained several Online Mendelian Inheritance in Man (OMIM) genes, including as coagulation factor VIII (F8), glucose-6-phosphate dehydrogenase (G-6-PD), inhibitor of nuclear factor- $\kappa \mathrm{B}$ kinase subunit $\gamma$ (IKBKG), trimethyllysine hydroxylase $\varepsilon$ (TMLHE), Ras-related protein Rab-39B (RAB39B) and chloride intracellular channel 2 (CLIC2). In addition, this derivative $\mathrm{X}$ chromosome contained the 12.329-Mb fragment from the Yq11.21-q11.23 interval of the
Y chromosome rather than the SRY gene. As the patient does not have SRY, he does not secrete testosterone and does not show male characteristics.

Prenatal diagnosis. Using karyotype analysis, FISH and SNP microarray assay, the number of this fetal chromosomes was determined as 46 , and the two sex chromosomes were $X$, of which one $\mathrm{X}$ chromosome was normal while the other one was unbalanced derivative $\mathrm{X}$ chromosome exhibiting complex inversion, translocation and deletion, notably exhibiting a pericentric inversion between Xp22.3 and Xq28 (one 1.241-Mb deletion in Xq28, including the OMIM genes, F8, G-6-PD, IKBKG, TMLHE, RAB39B and CLIC2). The derivative $\mathrm{X}$ chromosome also exhibited fragment translocation between Yq11.21 and q11.23 (not containing the SRY gene). Therefore, this fetus demonstrated inversion, translocation, and deletion (deletion/duplication) syndrome; therefore, it exhibited $\mathrm{X}$-linked recessive type A hemophilia and X-linked incomplete dominant favism simultaneously. Furthermore, as a result of a lack of the RAB39B and CLIC2 genes, this fetus may have experienced various degrees of cognitive impairment and developmental abnormalities in future. The novel derivative $\mathrm{X}$ chromosome contained a fragment in Yq11.21q11.23, although it did not contain the SRY gene; therefore, its social gender would have been female. However, whether this fragment may have impacted the growth and development of this fetus after birth remains unknown.

Pregnancy outcome and autopsy results. The two parents had normal karyotypes, but the fetal chromosomal abnormality was a novel chromosome derivation. As this derivative chromosome exhibited complex inversion, translocation and deletion, the genetic counseling expert informed the patient and her family of the corresponding clinical phenotypes after birth, and the certificate of prenatal diagnosis was issued in accordance with the Ethics Committee of the People's Hospital of Peking University. The patient and her family decided to terminate the pregnancy, and labor was induced at the 27 weeks and 4 days of gestation. The autopsy and pathological results identified that it was a female fetus, exhibiting a vulva, vagina, uterus and ovaries, and the appearance of the fetus was not abnormal. 
The ovarian pathological results included ovarian tissue cells and no testicular tissue components were observed.

\section{Discussion}

Different genetic testing methods have their advantages, disadvantages and applications $(1,2,6)$; therefore, prenatal diagnosis should be tailored to the characteristics of different cases and use comprehensive diagnostic methods to determine an accurate diagnosis. When the routine karyotyping analysis indicates novel structural aberration in suspicious chromosomes, site-specific probes should be designed to test the corresponding genes at the breakpoints of the structurally abnormal chromosome so as to confirm or rule out the chromosomal structural abnormalities. When the suspicious chromosome exhibits micro-fragment deletion or duplication, the gene microarray technique could be used to detect copy-number variants (10-12).

Therefore, in the present study, routine karyotype analysis of the case revealed that one $\mathrm{X}$ chromosome was a de novo derivative, which was abnormal and exhibited pericentric inversion and long arm translocation of the $\mathrm{Y}$ chromosome. The DAPI staining confirmed that this abnormal $\mathrm{X}$ chromosome contained a fragment from the Y chromosome. The FISH assay using the SRY/DXZ1 probe identified a negative SRY signal, confirming that the abnormal chromosome-translocated Y chromosome only had the long arm fragment instead of the testis-determining factor region in the short arm. The positive DXZ1 signal confirmed the centromere was from the $\mathrm{X}$ chromosome, notably this derivative chromosome was an $\mathrm{X}$ chromosome. The FISH assay using the XYpter (green)/XYqter (red) and STS probes confirmed the presence of the pericentric inversion in this abnormal $\mathrm{X}$ chromosome, and the FISH test using the RP11-64L19 probe (located in $\mathrm{Xq} 28$ ) confirmed the breakpoint of the chromosomal inversion was located before the Xq28 locus; thus, the inversion occurred between Xp22.3 and Xq28. Whole gene microarray detection of the fetus confirmed the deletion of one $1.241-\mathrm{Mb}$ fragment in q28 of the long arm of the $\mathrm{X}$ chromosome, as well as the fragment translocation between Yq11.21 and q11.23.

During the diagnostic process of the current case, G-banding karyotype analysis and DAPI staining only determined that this abnormal chromosome was derivative from the translocation of the X and Y chromosomes; if the FISH had not been performed, pericentric inversion and breakpoints in the abnormal chromosome would not have been observed. Furthermore, without performing the SNP microarray, neither the deletion at the distal end of $\mathrm{Xq} 28$, nor the fragment size containing the $\mathrm{Y}$ chromosome would not have been determined. Thus, only by comprehensively applying the various detection methods of karyotyping analysis, FISH or SNP were the nature, origin and manifestations of this chromosomal-derivative abnormalities established. This enabled the successful diagnosis of this case of unbalanced sex chromosomal inversion, translocation and deletion.

When one chromosome breaks during the 'hit' event, translocation, rearrangement and reconnection of different chromosomes occurs, thus forming a novel derivative chromosome during the repair process $(13,14)$. If the derivative chromosome exhibits fragment deletion or gene damage at the breakpoints, it's termed unbalanced translocation and/or inversion. The patients with unbalanced translocation and/or inversion would often exhibit the corresponding genetic effects due to gene loss or damage. As the formation of complex chromosomal translocation and/or inversion results from extrinsic factors acting on chromosomal exogenous DNA molecules, causing breakage, translocation, and reconnection of the chromosomes at the molecular level, men and women at childbearing ages should take measures to protect their reproductive organs when exposed to large doses of ionizing radiation or rays (15-17). The couple in the present study decorated their house before and during the early stages of pregnancy; therefore, contact with hazardous materials prior to pregnancy may have resulted in a 'hit' event in the parental germ cells (haploid chromosome) prior to fertilization or even after the father's sperm or the mother's egg were formed, thus the chromosome breakage, repair and reconnection occurred. The fetal derivative $\mathrm{X}$ chromosome only contained the partial long arm of the Y chromosome, while it did not contain the short arm that had the testis-determining genes; therefore, without the roles of male hormone testosterone, its genitalia would not differentiate into male, and its social gender would have been female. However, whether the translocated Y chromosome fragment would affect the growth and development of the fetus in future remains unknown. In addition, the long arm of the derivative $\mathrm{X}$ chromosome missed a 1.241-Mb fragment containing F8, G-6-PD, IKBKG, TMLHE, RAB39B, and CLIC2 (18-24). The F8 gene encodes the clotting factor VIII, therefore a homozygous mutation or deletion of this gene would cause X-linked recessive type A hemophilia; thus, the patient would be a type A hemophilia gene carrier. The mutation or deletion of the G-6-PD gene results in the deficiency of G-6-PD, thus exhibiting X-chain incomplete dominant 'favism'. It has been reported that mutations of the RAB39B and CLIC2 genes were associated with $\mathrm{X}$-linked mental retardation diseases, manifesting as mental retardation, developmental delay, congenital heart disease or epilepsy (25). It was also reported that the females carrying the CLIC2 mutation may exhibit mild cognitive impairment (26). Mutation of the DKC1 gene has been associated with the X-linked dyskeratosis congenita (27), manifesting as skin and mucosal abnormalities, progressive myelodysplasia or organ abnormalities. In addition, it was reported that the female DKC1-mutation carriers would exhibit increased risks of the above-mentioned diseases with variable phenotypic expression. Therefore, it could be hypothesized that certain $\mathrm{X}$-linked recessive genetic diseases may exhibit specific clinical phenotypes in female carriers, which may be due to the random $\mathrm{X}$ chromosome inactivation or haploinsufficiency. Thus, it could not be ruled out that the fetus in the present case may have been at risk of different degrees of cognitive impairment, as well as the above-mentioned diseases. This was a case of unbalanced chromosomal inversion, translocation and deletion. Due to the gene deletion or displacement effects at the breakpoints of the $\mathrm{X}$ chromosome, clinical manifestations, such as corresponding gonad and reproductive dysfunction, could not be ruled out. The parents had normal chromosomal karyotypes, and the abnormal chromosome of the fetus was a de novo derivative, therefore its recurrence risk would not be high; however, routine prenatal diagnosis, ultrasound or 
SNP microarray should be considered according to the regular examinations during the pregnancy period.

In conclusion, the karyotype analysis, FISH, and whole genome microarray were performed for the prenatal diagnosis of a high-risk pregnant woman, and the fetus was diagnosed with sex chromosomal inversion, translocation, and deletion, so the corresponding clinical phenotypes associated with this derivative unbalanced chromosome (of chromosomal deletion/duplication) may have presented after birth. The current study provided adequate genetic counseling to the patient and her family, and the family decided to terminate the pregnancy as the fetus would have been born with birth defects. The present study may provide guidance for future pregnancy and a healthy birth.

\section{References}

1. Cancer Genome Atlas Research Network, Ley TJ, Miller C, Ding L, Raphael BJ, Mungall AJ, Robertson A, Hoadley K, Triche TJ Jr, Laird PW, et al: Genomic and epigenomic landscapes of adult de novo acute myeloid leukemia. N Engl J Med 368: 2059-2074, 2013

2. Garcia-Manero G: Myelodysplastic syndromes: 2014 update on diagnosis, risk-stratification, and management. Am J Hematol 89: 97-108, 2014.

3. Kantarjian HM, Larson RA, Cortés JE, Deering KL and Mauro MJ: Current practices in the management of chronic myeloid leukemia. Clin Lymphoma Myeloma Leuk 13: 48-54, 2013.

4. Stilgenbauer S, Schnaiter A, Paschka P, Zenz T, Rossi M, Döhner K, Bühler A, Böttcher S, Ritgen M, Kneba M, et al: Gene mutations and treatment outcomes in chronic lymphocytic leukemia: Results from the CLL8 trial. Blood 123: 3247-3254, 2014.

5. Gibson SE, Luo J, Sathanoori M,Liao J, Surti U and Swerdlow SH: Whole-genome single nucleotide polymorphism array analysis is complementary to classical cytogenetic analysis in the evaluation of lymphoid proliferations. Am J Clin Pathol 141: 247-255, 2014

6. Xu X, Johnson EB, Leverton L, Arthur A, Watson Q, Chang FL, Raca G and Laffin JJ: The advantage of using SNP array in clinical testing for hematological malignancies-a comparative study of three genetic testing methods. Cancer Genet 206 317-326, 2013

7. Dougherty MJ, Wilmoth DM, Tooke LS, Shaikh TH, Gai X, Hakonarson $\mathrm{H}$ and Biegel JA: Implementation of high resolution single nucleotide polymorphism array analysis as a clinical test for patients with hematologic malignancies. Cancer Genet 204: 26-38, 2011.

8. Okada M, Suto Y, Hirai M, Shiseki M, Usami A, Okajima K, Teramura M, Mori N and Motoji T: Microarray CGH analyses of chromosomal $20 \mathrm{q}$ deletions in patients with hematopoietic malignancies. Cancer Genet 205: 18-24, 2012.

9. Shaffer LG, McGowan-Jordan J and Schmid M (eds): An International System for Human Cytogenomic Nomenclature. Cytogenetic and Genome Research, Basel, 2013.

10. Mullighan CG: The molecular genetic makeup of acute lymphoblastic leukemia. Hematology Am Soc Hematol Educ Program 2012: 389-396, 2012.

11. Kolquist KA, Schultz RA, Furrow A, Brown TC, Han JY, Campbell LJ, Wall M, Slovak ML, Shaffer LG and Ballif BC: Microarray-based comparative genomic hybridization of cancer targets reveals novel, recurrent genetic aberrations in the myelodysplastic syndromes. Cancer Genet 204: 603-628, 2011.
12. Heinrichs S, Li C and Look AT: SNP array analysis in hematologic malignancies: Avoiding false discoveries. Blood 115: 4157-4161, 2010.

13. Lee MY, Seo CS, Kim JY and Shin HK: Genotoxicity evaluation of Guibi-Tang extract using an in vitro bacterial reverse mutation assay, chromosome aberration assay, and in vivo micronucleus test. BMC Complement Altern Med 14: 215, 2014.

14. Jenderny J: Chromosome aberrations in a large series of spontaneous miscarriages in the German population and review of the literature. Mol Cytogenet 7: 38, 2014.

15. Tawn EJ, Curwen GB, Jonas P, Riddell AE, Hodgson L: Chromosome aberrations determined by sFISH and G-banding in lymphocytes from workers with internal deposits of plutonium. Int J Radiat Biol 92: 312-320, 2016.

16. Fucić A, Zeljezić D, Kasuba V, Kopjar N, Rozgaj R, Lasan R, Mijić A, Hitrec V and Lucas JN: Stable and unstable chromosome aberrations measured after occupational exposure to ionizing radiation and ultrasound. Croat Med J 48: 371-377, 2007.

17. Themis M, Garimberti E, Hill MA and Anderson RM: Reduced chromosome aberration complexity in normal human bronchial epithelial cells exposed to low-LET $\gamma$-rays and high-LET $\alpha$-particles. Int J Radiat Biol 89: 934-943, 2013.

18. http://omim.org/; http://genome.ucsc.edu/; https://decipher. sanger.ac.uk/ and https: //decipher.sanger.ac.uk/

19. Antonarakis SE, Kazazian HH and Tuddenham EG: Molecular etiology of factor VIII deficiency in hemophilia A. Hum Mutat 5: 1-22, 1995.

20. Tuddenham EG, Cooper DN, Gitschier J, Higuchi M, Hoyer LW, Yoshioka A, Peake IR, Schwaab R, Olek K, Kazazian HH, et al: Haemophilia A: Database of nucleotide substitutions, deletions, insertions and rearrangements of the factor VIII gene. Nucleic Acids Res 19: 4821-4833, 1991.

21. Kaplan M, Renbaum P, Levy-Lahad E, Hammerman C, Lahad A and Beutler E: Gilbert syndrome and glucose-6-phosphate dehydrogenase deficiency: A dose-dependent genetic interaction crucial to neonatal hyperbilirubinemia. Proc Nat Acad Sci USA 94: 12128-12132, 1997.

22. van Bruggen R, Bautista JM, Petropoulou T, de Boer M, van Zwieten R, Gómez-Gallego F, Belohradsky BH, Hartwig NG, Stevens D, Mason PJ and Roos D: Deletion of leucine 61 in glucose-6-phosphate dehydrogenase leads to chronic nonspherocytic anemia, granulocyte dysfunction, and increased susceptibility to infections. Blood 100: 1026-1030, 2002.

23. Giannandrea M, Bianchi V, Mignogna ML, Sirri A, Carrabino S, D'Elia E, Vecellio M, Russo S, Cogliati F, Larizza L, et al: Mutations in the small GTPase gene RAB39B are responsible for X-linked mental retardation associated with autism, epilepsy, and macrocephaly. Am J Hum Genet 86: 185-195, 2010.

24. Wilson GR, Sim JC, McLean C, Giannandrea M, Galea CA, Riseley JR, Stephenson SE, Fitzpatrick E, Haas SA, Pope K, et al: Mutations in RAB39B cause X-linked intellectual disability and early-onset Parkinson disease with $\alpha$-synuclein pathology. Am J Hum Genet 95: 729-735, 2014.

25. Leonard $\mathrm{H}$ and Wen $\mathrm{X}$ : The epidemiology of mental retardation: Challenges and opportunities in the new millennium. Ment Retard Dev Disabil Res Rev 8: 117-134, 2002.

26. Takano K, Liu D, Tarpey P, Gallant E, Lam A, Witham S, Alexov E, Chaubey A, Stevenson RE, Schwartz CE, et al: An $\mathrm{X}$-linked channelopathy with cariomegaly due to a CLIC2 mutation enhancing ryanodine receptor channel activity. Hum Mol Genet 21: 4497-4507, 2012.

27. Alder JK, Parry EM, Yegnasubramanian S, Wagner CL, Lieblich LM, Auerbach R, Auerbach AD, Wheelan SJ and Armanios M: Telomere phenotypes in females with heterozygous mutations in the dyskeratosis congenita 1 (DKC1) gene. Hum Mutat 34: 1481-1485, 2013.

This work is licensed under a Creative Commons Attribution-NonCommercial-NoDerivatives 4.0 International (CC BY-NC-ND 4.0) License. 